Iva Tendais*, Bárbara Figueiredo, Hernâni Gonçalves, João Bernardes, Diogo Ayres-de-Campos and Nuno Montenegro

\title{
Sex differences in the fetal heart rate variability indices of twins
}

\begin{abstract}
Aims: To evaluate the differences in linear and complex heart rate dynamics in twin pairs according to fetal sex combination [male-female (MF), male-male (MM), and female-female (FF)].
\end{abstract}

Methods: Fourteen twin pairs (6 MF, $3 \mathrm{MM}$, and $5 \mathrm{FF}$ ) were monitored between 31 and 36.4 weeks of gestation. Twenty-six fetal heart rate (FHR) recordings of both twins were simultaneously acquired and analyzed with a system for computerized analysis of cardiotocograms. Linear and nonlinear FHR indices were calculated.

Results: Overall, MM twins presented higher intrapair average in linear indices than the other pairs, whereas FF twins showed higher sympathetic-vagal balance. MF twins exhibited higher intrapair average in entropy indices and MM twins presented lower entropy values than FF twins considering the (automatically selected) threshold $r_{\mathrm{Lu}}$. MM twin pairs showed higher intrapair differences in linear heart rate indices than MF and FF twins, whereas FF twins exhibited lower intrapair differences in entropy indices.

Conclusions: The results of this exploratory study suggest that twins have sex-specific differences in linear and nonlinear indices of FHR. MM twins expressed signs of a more active autonomic nervous system and MF twins showed the most active complexity control system. These results suggest that fetal sex combination should be taken into consideration when performing detailed evaluation of the FHR in twins.

\footnotetext{
*Corresponding author: Iva Tendais, School of Psychology, University of Minho, Campus de Gualtar, 4710-057 Braga, Portugal, E-mail: id1814@alunos.uminho.pt

Bárbara Figueiredo: School of Psychology, University of Minho, Braga, Portugal

Hernâni Gonçalves: Center for Research in Health Technologies and Information Systems (CINTESIS), Faculty of Medicine, University of Porto, Porto, Portugal

João Bernardes and Diogo Ayres-de-Campos: Institute of Biomedical Engineering, Faculty of Medicine, University of Porto, Sao Joao Hospital, Porto, Portugal

Nuno Montenegro: Faculty of Medicine, University of Porto, Sao Joao Hospital, Porto, Portugal
}

Keywords: Cardiotocography; entropy; fetal monitoring; spectral analysis; twins.

DOI 10.1515/jpm-2014-0031

Received January 29, 2014. Accepted May 23, 2014. Previously published online June 19, 2014.

\section{Introduction}

Consistent sex differences in health, behavior, and developmental outcomes after birth have been reported, but studies carried out during fetal life have yielded conflicting results. Previous studies on fetal heart rate (FHR) characteristics have reported sex-specific differences $[2-4,7,9$, $12]$, as well as no significant differences $[5,6,8,14,17,20]$, even when the same methodologies were used. In adults, women reveal a preponderance of vagal over sympathetic responsiveness in heart rate variability under basal conditions, especially before the age of $50[13,18,22,25]$. One possible explanation for sex differences in the autonomic nervous system is the effect of sex-specific hormones [13].

Studies in twins provide a unique setting to examine sex-related differences in FHR patterns. A variation in the intrauterine hormonal environment has been consistently found in litter-bearing mammals according to the sex of the adjacent fetuses [23], and a small number of studies in human twins also support the existence of sex hormone transfer between fetuses [16, 24], even though hormonal effects appear to be less strong [23]. To date, few studies have evaluated the sex differences in FHR in human twins [26].

The aim of this study was to evaluate the differences in linear and complex heart rate dynamics in twin pairs according to fetal sex combination: male-male (MM), male-female (MF), and female-female (FF).

\section{Methods}

The study protocol was approved by the local Ethics Committee and written informed consent was obtained from pregnant women prior to study enrollment. The study sample comprised 14 twin pairs (6 MF, $3 \mathrm{MM}$, and 
5 FF) from which 26 FHR recordings were simultaneously acquired in the antepartum period between 31 and 36.4 weeks of gestation. The average tracing length was $52.6 \mathrm{~min}$ (standard deviation, $\mathrm{SD}=45.2$ ) and the average \pm SD signal loss was $11.6 \% \pm 9.5 \%$. Between one and three tracings of each twin pair were available for analysis such that, in total, 13 tracings were from MF, 5 from MM, and 8 from FF. Three sets of twins were monochorionic (MC) (one monoamiotic and two diamniotic) and eleven were dichorionic (DC). Chorionicity was determined during routine ultrasound scan at 11-13 weeks of gestation [19] and confirmed by placental histology after birth. Pregnant women had an average of 29.4 years ( $\mathrm{SD}=4.1$, range $24-39$ years) and $42.9 \%$ had pregnancy complications such as preeclampsia, gestational diabetes, and premature rupture of membranes. Mean gestational age at delivery was 37.0 weeks ( $\mathrm{SD}=1.7$, range 32.1-38.7) and 43.1\% delivered before 37 weeks of gestation. Mean newborn birth weight was 2478.8 $\pm 442.7 \mathrm{~g}$ (range 1395-3290 g) and $42.0 \%$ had birth weight below $2500 \mathrm{~g}$. All newborns had normal 5-min Apgar scores, and only two second twins had a 1-min Apgar score below 7 . Admission to a neonatal intensive care unit occurred in $22 \%$.

The FHR monitoring of both twins was performed using the Philips Medical 50A fetal monitor (Philips Medical, Eindhoven, The Netherlands) connected by cable to the Omniview-SisPorto ${ }^{\circledR} 3.5$ system (Speculum, Lisbon, Portugal) for computer analysis of cardiotocograms [1]. A preprocessing algorithm was applied to reduce noise and artifacts as described elsewhere [11]. Additionally, persistent segments of signal loss in the initial period of the tracings were visually identified and excluded.

Linear and nonlinear FHR analysis were performed in the initial four 5-min segments of each tracing, after the exclusion of initial periods with signal loss. Time-domain linear analysis included mean FHR (mFHR), standard deviation of FHR (sdFHR), long-term irregularity (LTI), Delta FHR $(\Delta)$, short-term variation (STV), and interval index (II). Frequency domain (spectral) linear analysis included very low frequency (VLF), low frequency (LF), movement frequency (MF), and high frequency (HF). LF and HF are mainly associated with the influence of the sympathetic and parasympathetic branches of the autonomic nervous system, respectively, on heart rate variability (HRV). MF reflects fetal movements, as well as maternal breathing. LF/HF and LF/ $(\mathrm{MF}+\mathrm{HF})$ reflect sympathetic-vagal balance, and was also considered.

For nonlinear analysis, approximate entropy (ApEn) and sample entropy (SampEn) indices were computed, considering the embedding dimension $(m)$ equal to 2 and the values 0.1 SD, 0.15 SD, 0.2 SD, and $r_{\mathrm{Lu}}$ [15] for the threshold parameter $(r)$. These indices measure the complexity and irregularity of time series and have been linked to complex cortical nervous system activity [21]. Short- and long-term variation were also assessed by the SD1 and SD2 indices, respectively, extracted from the Poincaré plot analysis, as well as the SD1/SD2 ratio.

Linear and nonlinear indices were calculated as described elsewhere $[10,11]$. Intrapair average $[(A+B) / 2]$ and absolute differences $(|A-B|)$ for each calculated FHR index, between MF, MM and FF twins, were evaluated using $95 \%$ bootstrap $(B=1000)$ percentile confidence intervals (CIs) (95\% CI) for the median, and nonparametric Mann-Whitney and Kruskal-Wallis statistical tests, setting significance at $\mathrm{P}<0.05$. Data were analyzed with IBM SPSS 19 Windows version (PASW Statistics for Windows, SPSS Inc., Chicago, IL, USA).

\section{Results}

Male-male twins presented a significantly higher intrapair average of time-domain linear FHR indices than the others, as well as for the spectral indices VLF, LF, MF, and HF. A significantly lower sympathetic-vagal balance of MM twins was also observed through $\mathrm{LF} /(\mathrm{MF}+\mathrm{HF})$. The intrapair average of entropy indices with the threshold values $0.1,0.15$, and 0.2 was consistently found to be decreasing from MF, MM and FF twins, but significance was only found between MF and FF, whereas MMs presented significantly lower entropy values considering the threshold $r_{\text {Lu }}$. Short- and long-term variations as measured by indices SD1 and SD2, respectively, were significantly higher for MM twins, and the ratio SD1/SD2 decreased significantly with declining numbers of male fetuses.

Intrapair absolute differences of sdFHR, $\Delta$, and STV indices were significantly higher between MM twins than between the others. Moreover, these differences were more pronounced as the number of male fetuses in the pair increased. Similar results were achieved with spectral indices VLF, LF, MF and HF, as well as for SD1 and SD2 variation indices of the Poincaré plot. The intrapair absolute differences of ApEn and SampEn were significantly lower between FF twins than between MF or MM twins. The intrapair average and absolute difference for each FHR index are presented in Table 1. The evolution of FHR indices STV, $\mathrm{LF} /(\mathrm{MF}+\mathrm{HF})$, and $\operatorname{SampEn}\left(2, r_{\mathrm{Lu}}\right)$, as a function of the gestational age, is shown in Figure 1.

The three MC FF pregnancies were compared with two DC FF twins. In general, both intrapair averages and absolute differences were similar, with the exception of a significantly $(\mathrm{P}=0.013)$ lower II average and lower $(\mathrm{P}=0.041)$ absolute difference of $\mathrm{LF} / \mathrm{HF}$ in $\mathrm{MC}$ pregnancies.

\section{Discussion}

The FHR indices were significantly influenced by sex combination in twins, both in intrapair average and absolute differences. Previous research in singleton pregnancies already indicated that fetal sex significantly influences FHR indices [2, 3].

In general, $\mathrm{MM}$ twins presented higher linear indices than FF pairs, a finding in accordance with the previous results, and this may be related with a higher predominance of behavioral FHR pattern B (active sleep) in male fetuses [2]. The MM twins also presented higher short- and long-term variability than others. These findings are in line with the results of singleton studies showing a more active autonomous nervous system [2] and a higher FHR variability in male fetuses [7]. A lower sympathetic-vagal balance was observed in MM twins than in FF. Although MM twins consistently presented a higher intrapair 
Table 1 Bootstrap percentile $95 \% \mathrm{Cls}$ for the intrapair average $[(A+B) / 2]$ and intrapair absolute differences $(|A-B|)$ of linear and nonlinear FHR indices for the MF, MM, and FF groups.

\begin{tabular}{|c|c|c|c|c|c|c|c|c|}
\hline & \multicolumn{4}{|r|}{$(A+B) / 2$} & \multicolumn{4}{|r|}{$\mid A-B$} \\
\hline & MF & MM & $\mathbf{F F}$ & P-values & MF & MM & $\mathbf{F F}$ & P-values \\
\hline $\mathrm{mFHR}$ & $137.39-144.54$ & $145.66-151.30$ & $138.96-147.59$ & $a, b, d$ & $5.10-7.91$ & $2.02-5.98$ & $3.67-6.02$ & b \\
\hline sdFHR & $4.92-6.40$ & $6.70-9.12$ & $6.09-8.04$ & $\mathrm{a}, \mathrm{b}, \mathrm{c}$ & $1.45-3.02$ & $2.76-6.63$ & $0.77-3.06$ & $a, b, d$ \\
\hline LTI & $6.69-9.59$ & $9.43-14.26$ & $9.64-14.10$ & $\mathrm{a}, \mathrm{b}, \mathrm{c}$ & $2.03-4.69$ & $1.25-8.71$ & $2.16-5.30$ & \\
\hline$\Delta$ & $17.56-21.38$ & $24.66-30.89$ & $18.05-25.75$ & $a, b, d$ & $3.98-7.85$ & $8.38-24.30$ & $2.40-9.20$ & $\mathrm{a,b,d}$ \\
\hline STV & $2.22-2.65$ & $3.26-4.06$ & $2.14-3.15$ & $a, b, d$ & $0.62-1.05$ & $1.02-2.82$ & $0.26-0.52$ & $\mathrm{a}, \mathrm{b}, \mathrm{c}, \mathrm{d}$ \\
\hline II & $0.44-0.47$ & $0.40-0.51$ & $0.36-0.42$ & $\mathrm{a}, \mathrm{c}, \mathrm{d}$ & $0.06-0.15$ & $0.07-0.11$ & $0.04-0.10$ & \\
\hline TP & $16.53-26.83$ & $35.59-62.67$ & $14.70-37.97$ & $\mathrm{a}, \mathrm{b}, \mathrm{d}$ & $7.40-18.66$ & $24.84-83.87$ & $4.61-20.09$ & $a, b, d$ \\
\hline VLF & $6.27-11.84$ & $12.10-17.92$ & 7.33-15.39 & $a, b$ & $3.05-10.06$ & $8.48-21.88$ & $2.60-7.36$ & $a, b, d$ \\
\hline LF & $6.90-12.30$ & $18.69-42.4$ & $7.41-18.64$ & $\mathrm{a}, \mathrm{b}, \mathrm{d}$ & $3.38-8.54$ & $12.06-64.43$ & $2.56-5.28$ & $a, b, d$ \\
\hline MF & $0.73-1.42$ & $3.07-5.50$ & $0.61-1.73$ & $a, b, d$ & $0.44-0.90$ & $3.49-6.68$ & $0.21-0.94$ & $a, b, d$ \\
\hline $\mathrm{HF}$ & $0.21-0.35$ & $0.67-1.16$ & $0.17-0.31$ & $\mathrm{a}, \mathrm{b}, \mathrm{d}$ & $0.10-0.20$ & $0.54-1.40$ & $0.07-0.13$ & $a, b, d$ \\
\hline $\mathrm{LF} /(\mathrm{MF}+\mathrm{HF})$ & $6.17-8.32$ & $4.89-6.95$ & $6.38-10.37$ & $\mathrm{a}, \mathrm{b}, \mathrm{d}$ & $2.70-4.07$ & $0.98-3.31$ & $1.85-4.92$ & \\
\hline $\mathrm{LF} / \mathrm{HF}$ & $32.82-50.43$ & $23.02-44.62$ & $35.15-61.88$ & d & $13.53-36.13$ & $10.17-25.47$ & $16.02-45.30$ & \\
\hline $\operatorname{ApEn}(2,0.1)$ & $0.74-0.82$ & $0.61-0.83$ & $0.67-0.74$ & c & $0.13-0.26$ & $0.12-0.24$ & $0.09-0.21$ & \\
\hline $\operatorname{ApEn}(2,0.15)$ & $0.60-0.67$ & $0.47-0.70$ & $0.49-0.55$ & $\mathrm{a}, \mathrm{c}$ & $0.13-0.25$ & $0.13-0.26$ & $0.08-0.18$ & \\
\hline $\operatorname{ApEn}(2,0.2)$ & $0.44-0.53$ & $0.39-0.56$ & $0.36-0.42$ & $a, c$ & $0.14-0.19$ & $0.11-0.21$ & $0.05-0.14$ & $a, c, d$ \\
\hline $\operatorname{ApEn}\left(2, r_{\mathrm{Lu}}\right)$ & $0.81-0.89$ & $0.64-0.83$ & $0.79-0.86$ & $\mathrm{a}, \mathrm{b}, \mathrm{d}$ & $0.10-0.19$ & $0.08-0.24$ & $0.04-0.18$ & \\
\hline SampEn $(2,0.1)$ & $0.64-0.73$ & $0.48-0.66$ & $0.55-0.63$ & $\mathrm{a}, \mathrm{b}$ & $0.17-0.32$ & $0.10-0.28$ & $0.11-0.21$ & \\
\hline SampEn(2, 0.15) & $0.48-0.56$ & $0.35-0.53$ & $0.37-0.44$ & $\mathrm{a}, \mathrm{c}$ & $0.13-0.25$ & $0.10-0.22$ & $0.09-0.19$ & \\
\hline SampEn(2, 0.2) & $0.35-0.42$ & $0.28-0.42$ & $0.28-0.32$ & $\mathrm{a}, \mathrm{c}$ & $0.12-0.19$ & $0.09-0.21$ & $0.05-0.12$ & $a, c, d$ \\
\hline $\operatorname{SampEn}\left(2, r_{\mathrm{Lu}}\right)$ & $0.71-0.82$ & $0.48-0.66$ & $0.66-0.82$ & $a, b, d$ & $0.15-0.28$ & $0.10-0.30$ & $0.09-0.31$ & \\
\hline SD1 & $0.68-0.93$ & $1.21-1.62$ & $0.66-0.89$ & $a, b, d$ & $0.15-0.29$ & $0.49-1.18$ & $0.11-0.25$ & $a, b, d$ \\
\hline SD2 & $6.93-9.00$ & $9.41-12.95$ & $8.59-11.35$ & $a, b, c$ & $1.99-4.37$ & $3.37-9.23$ & $1.04-4.35$ & $a, b, d$ \\
\hline SD1/SD2 & $0.10-0.11$ & $0.11-0.14$ & $0.07-0.09$ & $\mathrm{a}, \mathrm{c}, \mathrm{d}$ & $0.03-0.04$ & $0.01-0.04$ & $0.01-0.05$ & \\
\hline
\end{tabular}

${ }^{\mathrm{a} P}<0.05 \mathrm{MF}$ vs. $\mathrm{MM}$ vs. $\mathrm{FF} ;{ }^{b} \mathrm{P}<0.05 \mathrm{MF}$ vs. $\mathrm{MM}$; ${ }^{\mathrm{C}}<<0.05 \mathrm{MF}$ vs. $\mathrm{FF} ;{ }^{\mathrm{d}} \mathrm{P}<0.05 \mathrm{MM}$ vs. FF.

average of spectral indices, FF twins showed a marked decrease in parasympathetic activity (measured by the HF component), thus leading to an increase in the sympathetic-vagal balance. The latter was accomplished by higher intrapair absolute differences in MM twins. Similar results were obtained for the indices total power and SD1/ SD2. Although a similar trend was previously found in the singleton fetuses [2], these findings contrast with those reported in the majority of studies in adults [13, 18, 22].

Consistent with findings from singletons [2], FF pairs exhibited more complex FHR activity than MM twins as measured by $\mathrm{ApEn}_{\mathrm{Lu}}$ and $\mathrm{SampEn}_{\mathrm{Lu}}$ suggesting a more active complexity control system. However, no differences were found between FF and MM twin pairs in the other entropy indices. This inconsistency may indicate that signal complexity is not being captured when the recommended values for tolerance threshold (0.1-0.2 SD of the data) are applied. Nevertheless, MF pairs showed a consistently higher complexity of FHR data as measured by all entropy indices. The reasons for this result are not clear. Future studies comparing heart rate dynamics between males and females from same and opposite-sex twin pairs are, therefore, recommended.

The largest intrapair absolute differences were observed in MM twins for most linear indices, as well as in the short- (SD1) and long-term (SD2) variability measured by the Poincaré plot. This suggests a lower synchronization in the activity of MM twins. The mFHR index was an exception, where MF pairs presented the largest intrapair differences, probably reflecting a consistently higher mFHR in MMs and lower mFHR in FFs. MFs tended to be closer to FFs than to MMs in linear indices, while the opposite occurred for nonlinear indices. These findings are somewhat unexpected and suggest that factors other than sex may contribute to HRV. If fetal sex was to be a major factor to HRV, then MF would have the largest intrapair differences, and no difference would be found between the same-sex twin pairs. Several factors could explain the differential effect of sex combination on linear and nonlinear indices, such as fetal hormone transfer, fetal presentation, and intertwins contacts. We speculate that the largest intrapair differences in MM pairs may be due to higher male reactivity 

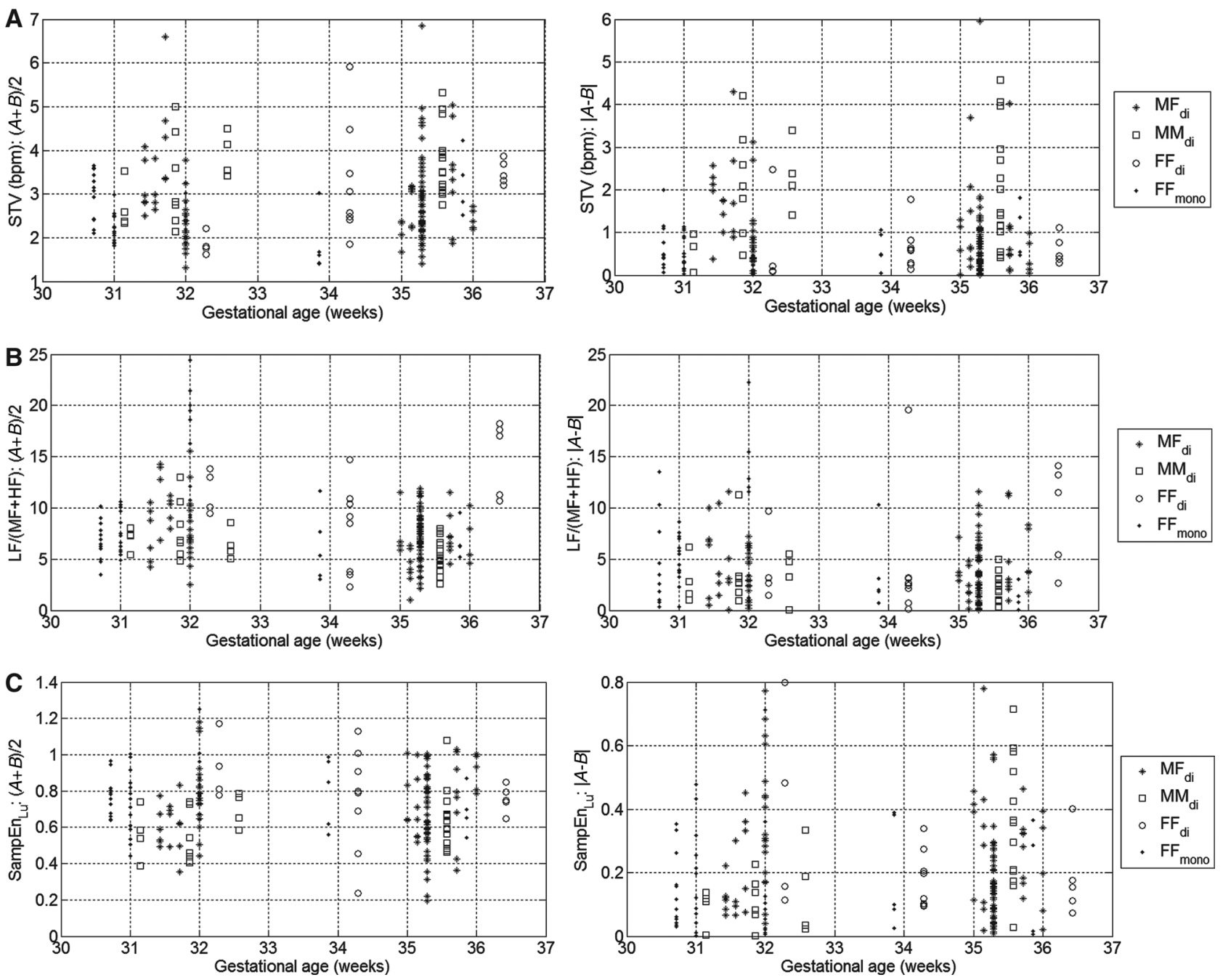

Figure 1 Evolution of FHR indices (A) STV, (B) LF/(MF+HF), and (C) SampEn $\left(2, r_{\mathrm{Lu}}\right)$ as a function of the gestational age, with respect to the twin pairs MF (DC), MM (DC), and FF (DC and MC). Left plots: intrapair average, $(A+B) / 2$; right plots: absolute differences $|A-B|$.

associated with their increased autonomic activity, as well as to intrapair differences in presentation, as most MM had cephalic/breech presentation and breech presentations may had been more exposed to the noise produced by the maternal heart rate and gastric movements (Bernardes et al., unpublished observations). The smallest intrapair differences in FFs are probably related to the inclusion of more MC twins in this group. Chorionicity has been shown to influence FHR patterns with MC twins showing more similarities in FHR patterns than DC twins [26].

The automated analysis of cardiotocograms is a major strength of this study given its objectivity, reproducibility, and predictive validity. Conversely, the visual interpretation of the tracings has high inter- and intraobserver variability. Nevertheless, several limitations to this study need to be acknowledged, namely the small sample size and the inability to control for other possible confounders.
The results of this exploratory study suggest that fetal sex needs to be taken into consideration when performing a detailed evaluation of HRV in twins, as significant differences in heart rate dynamics may be found according to sex combination. Further research is needed to evaluate the influence of gestational age, type of presentation, and fetal behavior state on FHR indices, in line with the previous research in singletons. This may help to further clarify the complex relations that exist between twins and improve perinatal care in twin pregnancies.

Acknowledgments: This work was supported by a grant (SFRH/BD/40146/2007) to the first author from Fundação para a Ciência e Tecnologia. Hernâni Gonçalves is financed by a postdoctoral grant (SFRH/BPD/69671/2010) from the Fundação para a Ciência e a Tecnologia (FCT), Portugal. 


\section{References}

[1] Ayres-Campos D, Sousa P, Costa A, Bernardes J. Omniview-SisPorto 3.5 - a central fetal monitoring station with online alerts based on computerized cardiotocogram+ST event analysis. J Perinat Med. 2008;36:260-4.

[2] Bernardes J, Gonçalves H, Ayres-de-Campos D, Rocha AP. Linear and complex heart rate dynamics vary with sex in relation to fetal behavioural states. Early Hum Dev. 2008;84:433-9.

[3] Bernardes J, Gonçalves H, Ayres-de-Campos D, Rocha AP. Sex diferences in linear and complex fetal heart rate dynamics of normal and acidemic fetuses in the minutes preceding delivery. J Perinat Med. 2009;37:168-76.

[4] Buss C, Davis EP, Class QA, Gierczak M, Pattillo C, Glynn LM, et al. Maturation of the human fetal startle response: evidence for sex-specific maturation of the human fetus. Early Hum Dev. 2009;85:633-8.

[5] Dawes NW, Dawes GS, Moulden M, Redman CW. Fetal heart rate patterns in term labor vary with sex, gestational age, epidural analgesia, and fetal weight. Am J Obstet Gynecol. 1999;180:181-7.

[6] DiPietro JA, Caulfield L, Costigan KA, Merialdi M, Nguyen RH, Zavaleta N, et al. Fetal neurobehavioral development: a tale of two cities. Dev Psychol. 2004;40:445-56.

[7] DiPietro JA, Costigan KA, Shupe AK, Pressman EK, Johnson TR. Fetal neurobehavioral development: associations with socioeconomic class and fetal sex. Dev Psychobiol. 1998;33:79-91.

[8] DiPietro JA, Hodgson DM, Costigan KA, Hilton SC, Johnson TR. Development of fetal movement-fetal heart rate coupling from 20 weeks through term. Early Hum Dev. 1996;44:139-51.

[9] Fleisher LA, Dipietro JA, Johnson TR, Pincus S. Complementary and non-coincident increases in heart rate variability and irregularity during fetal development. Clin Sci (Lond). 1997;92:345-9.

[10] Gonçalves H, Bernardes J, Ayres-de-Campos D. Gender-specific heart rate dynamics in severe intrauterine growth-restricted fetuses. Early Hum Dev. 2013;89:431-7.

[11] Gonçalves H, Rocha AP, Ayres-de-Campos D, Bernardes J. Linear and nonlinear fetal heart rate analysis of normal and acidemic fetuses in the minutes preceding delivery. Med Biol Eng Comput. 2006;44:847-55.

[12] Groome LJ, Mooney DM, Holland SB, Smith LA, Atterbury JL, Dykman RA. Behavioral state affects heart rate response to low-intensity sound in human fetuses. Early Hum Dev. 1999;54:39-54.

[13] Kuo TB, Lin T, Yang CC, Li CL, Chen CF, Chou P. Effect of aging on gender differences in neural control of heart rate. Am J Physiol Heart Circ Physiol. 1999;277:H2233-9.
[14] Lange S, Van Leeuwen P, Geue D, Hatzmann W, Gronemeyer D. Influence of gestational age, heart rate, gender and time of dayon fetal heart rate variability. Med Biol Eng Comput. 2005;43:481-6.

[15] Lu S, Chen X, Kanters JK, Solomon IC, Chon KH. Automatic selection of the threshold value $r$ for approximate entropy. IEEE Trans Biomed Eng. 2008;55:1966-72.

[16] McFadden D, Loehlin JC, Pasanen EG. Additional finding on heritability and prenatal masculinization of cochlear mechanisms: click-evoked otoacoustic emissions. Hear Res. 1996;97:102-19.

[17] McKenna DS, Ventolini G, Neiger R, Downing C. Gender-related differences in fetal heart rate during first trimester. Fetal Diagn Ther. 2006;21:144-7.

[18] Mendonça GV, Heffernan KS, Rossow L, Guerra M, Pereira FD, Fernhall B. Sex differences in linear and nonlinear heart rate variability during early recovery from supramaximal exercise. Appl Physiol Nutr Metab. 2010;35:439-6.

[19] Montenegro N, Matias A. First trimester ultrasound. In: Kurjak A, Chervenak FA, editors. Textbook of Perinatal Medicine. 2nd ed. New York: Parthenon Publishing; 2006. p. 1053-63.

[20] Oguch O, Steer P. Gender does not affect fetal heart rate variation. Br J Obstet Gynaecol. 1998;105:1312-4.

[21] Pincus S, Viscarello R. Approximate entropy: a regularity measure for fetal heart rate analysis. Obstet Gynecol. 1992;79:249-55.

[22] Ramaekers D, Ector H, Aubert AE, Rubens A, van de Werf F. Heart rate variability and heart rate in healthy volunteers: is the female autonomous nervous system cardioprotective? Eur Heart J. 1993;19:1334-41.

[23] Ryan BC, Vandenbergh JG. Intrauterine position effects. Neurosci Biobehav Rev. 2002;26:665-78.

[24] Shinwell ES, Reichman B, Lerner-Geva L, Boyko V, Blickstein I, Israel Neonatal Network. "Masculinizing" effect on respiratory morbidity in girls from unlike-sex preterm twins: a possible transchorionic paracrine effect. Pediatrics. 2007;120:e447-53.

[25] Sztajzel J, Jung M, Bayes de Luna A. Reproducibility and gender related differences of heart rate variability during all-day activity in young men and women. Ann Noninvasive Electrocardiol. 2008;13:270-7.

[26] Tendais I, Visser GHA, Figueiredo B, Montenegro N, Mulder EJH. Fetal behavior and heart rate in twin pregnancy: a review. Twin Res Hum Genet. 2013;14:1-10.

The authors stated that there are no conflicts of interest regarding the publication of this article. 